\title{
El parto prematuro se asocia a una menor sobrevida en la infancia y a una menor reproducción
}

Preterm birth is associated with less survival in childhood and less reproduction

\section{Objetivo}

Determinar los efectos del parto prematuro sobre la sobrevida en largo plazo, la reproducción y la probabilidad de parto prematuro en la siguiente generación.

\section{Diseño}

Observacional longitudinal de base poblacional llevado a cabo en Noruega.

\section{Pacientes y métodos}

En el Registro Médico de Nacimientos de Noruega fueron recabados datos sobre 1167506 nacimientos únicos entre 1967 y 1988. La cohorte fue seguida hasta 2002 para evaluar la sobrevida y fue "truncada" para los nacimientos ocurridos entre 1967 y 1976 para evaluar el nivel educacional y el resultado reproductivo hasta el año 2004.

Tabla 1: tasa de mortalidad durante la niñez y tasa de reproducción en nacidos prematuros.

\begin{tabular}{|c|c|c|c|c|}
\hline \multirow{2}{*}{\multicolumn{2}{|c|}{ Prematuros (semanas) }} & Nifiez temprana & Nifiez tardía & \multirow{2}{*}{$\begin{array}{r}\text { Reproducción } \\
\text { (BR; IC95\%) }\end{array}$} \\
\hline & & Mortalidad (BR; IC95\%) & Mortalidad (RR; IC95\%) & \\
\hline \multirow{2}{*}{22 a 27} & Niños & $1,33 \%(5,3 ; 2,0$ a 14,2$)$ & $1,01 \%(7,0 ; 2,3$ a 22,0$)$ & $13,9 \%(0,24 ; 0,17$ a 0,32$)$ \\
\hline & Niñas & $1,71 \%(9,7 ; 4,0$ a 23,7$)$ & $0 \%$ & $25 \%(0,33 ; 0,26$ a 0,42$)$ \\
\hline \multirow{2}{*}{28 a 32} & Niños & $0,73 \%(2,5 ; 1,6$ a 3,7$)$ & $0,37 \%(2,3 ; 1,3$ a 4,1$)$ & $38,6 \%(0,7 ; 0,66$ a 0,74$)$ \\
\hline & Niñas & $0 \%$ & $0 \%$ & $59,2 \%(0,81 ; 0,78$ a 0,85$)$ \\
\hline
\end{tabular}

\section{Conclusión}

En personas nacidas en Noruega entre 1967 y 1988, el parto prematuro se asoció a una menor sobrevida a lo largo de la infancia y a una menor tasa de reproducción.

\section{Medición de resultados principales}

Fueron evaluados el sexo, la edad gestacional al nacimiento, la mortalidad absoluta, el riesgo de muerte (fetal, neonatal, infantil y adolescente) y la incidencia y el riesgo de reproducir un parto prematuro en la generación siguiente. Los embarazos de término ( 37 a $42 \mathrm{sem}$ ) las muertes fetales y los recién nacidos vivos, estratificados por sexo, sirvieron como grupo de referencia para el análisis.

\section{Resultados}

La prematurez fue mayor en varones $(5,6 \%)$ que en niñas $(4,7 \%)$. Los nacidos prematuros presentaron un incremento de la mortalidad a lo largo de toda la infancia y menor reproducción. Ver tabla 1. Las mujeres pretérmino pero no los varones mostraron un mayor riesgo de tener un hijo prematuro.

\section{Comentario}

El presente trabajo muestra el impacto del parto prematuro en el largo plazo, evidenciando que tanto la mortalidad temprana como la tardía, especialmente en varones, es mas elevada en este grupo. Por otro lado también se observa una caída en la tasa de reproducción así como un incremento en el riesgo de que se produzca un parto prematuro en la siguiente generación. Lo importante de este estudio es que reproduce hallazgos de estudios anteriores con muestras más acotadas (hospitalarias o de algunas redes de información) ${ }^{1}$ ahora en una población general muy extensa (Noruega). Por otro lado, la rigurosidad de la recolección de los datos, la baja tasa de emigración y la cobertura uniforme de salud son ventajas que consolidan el trabajo de estos investigadores.
Palabras claves: parto prematuro, pronóstico, sobrevida, reproducción. Key words: premature birth, prgnosis, suvival, reproduction.

Fuente de financiamiento: no referida.
Las preguntas que se generan son si este efecto es completamente biológico o ambiental; esto último sustentado en un menor nivel educativo (estadísticamente no significativo) entre los prematuros. Por otro lado, esta cohorte es antigua (1967 a 1988) y han ocurrido enormes cambios en el cuidado prenatal y postnatal de estos niños, que han modificado las tasas de sobrevida y la calidad de la misma².

\section{Conclusión del comentador}

La rigurosidad de este trabajo, consistente con investigaciones previas, confirma que el parto prematuro se asocia a una menor sobrevida en la infancia y a una menor reproducción.

Carlos Fustiñana [ Jefe de Servicio de Neonatología. Hospital Italiano de Buenos Aires. carlos.fustiñana@hospitalitaliano.org.ar ]

Fustiñana C. El parto prematuro se asocia a una menor sobrevida en la infancia y a una menor reproducción. Evid. Actual. Práct. Ambul; $12(1): 16$. Ene-Mar 2009. Comentado de: Swamy G y col. Association of preterm birth with long-term survival, reproduction, and next-generation preterm birth. JAMA. 2008 Mar 26;299(12):1429-36. PMID: 18364485.

\section{Referencia}

1. Haack M y col. Outcomes in young adulthood for VLBWI. N Engl J Med 200;346(3): 149-157.

2. Fustiñana $\mathrm{C}$ y col. Medición de la efectividad en la evaluación de un programa de Cuidado intensivo neonatal. Impacto del uso de surfactante. Arch Argent Pediatr 2009;107(1):9-15. 